\title{
Effective properties of a poroelastic medium containing a distribution of aligned cracks
}

\author{
R. J. Galvin ${ }^{1}$ and B. Gurevich ${ }^{1,2}$ \\ Received 25 August 2008; revised 2 March 2009; accepted 14 May 2009; published 15 July 2009.
}

[1] We simulate the effect of fractures by considering them to be thin circular cracks in a poroelastic background. Using the solution of the scattering problem for a single-crack and multiple-scattering theory, we estimate the attenuation and dispersion of elastic waves in a porous medium containing a sparse distribution of cracks. When comparing with a similar model, in which multiple-scattering effects are neglected, we find that there is agreement at high frequencies and discrepancies at low frequencies. We conclude that the interaction between cracks should not be neglected at low frequencies, even in the limit of weak crack density. Since the models only agree with each other at high frequencies, when the time available for fluid diffusion is small, we conclude that the interaction between cracks, which is a result of fluid diffusion, is negligible at high frequencies. We also compare our results with a model for spherical inclusions and find that the attenuation for spherical inclusions has exactly the same dependence upon frequency but a difference in magnitude, which depends upon frequency. Since the attenuation curves are very close at low frequencies, we conclude that the effective medium properties are not sensitive to the shape of an inclusion at wavelengths that are large compared with the inclusion size. However, at frequencies such that the wavelength is comparable to or smaller than the inclusion size, the effective properties are sensitive to the greater compliance of the flat cracks, and more attenuation occurs at a given frequency as a result.

Citation: Galvin, R. J., and B. Gurevich (2009), Effective properties of a poroelastic medium containing a distribution of aligned cracks, J. Geophys. Res., 114, B07305, doi:10.1029/2008JB006032.

\section{Introduction}

[2] Naturally fractured reservoirs are becoming increasingly important for oil and gas exploration in many areas of the World. Because fractures may control the permeability of a reservoir it is important to be able to find and characterize fractured zones. In order to characterize a fractured reservoir we need to understand the effect the fractures will have on its overall elastic properties. Fractures are highly compliant compared to the relatively stiff pores, so fluid will flow between pores and fractures during passage of the seismic wave. In essence, a rock containing both pores and fractures can be thought of as a particular case of a dual-porosity material [Berryman and Wang, 1995; Auriault and Boutin, 1994]. If the fractures are aligned, the reservoir will exhibit long wavelength effective anisotropy. Since the fluid flow and scattering caused by the presence of

\footnotetext{
${ }^{1}$ CSIRO Division of Petroleum Resources, Perth, Western Australia, Australia.

${ }^{2}$ Department of Exploration Geophysics, Curtin University of Technology, Perth, Western Australia, Australia.

Copyright 2009 by the American Geophysical Union. 0148-0227/09/2008JB006032\$09.00
}

fractures depends upon seismic frequency, the anisotropy will be frequency dependent.

[3] In the limit of low frequencies static models can be used to obtain the effective elastic moduli of the fluidsaturated medium in terms of the properties of the dry skeleton and the saturating fluid [Gassmann, 1951; Brown and Korringa, 1975; Thomsen, 1995; Gurevich, 2003; Cardona, 2002]. For these models to be valid, fluid pressure must have time to fully equilibrate throughout the connected porespace which will only be the case at low frequencies. At higher frequencies pressure equilibration will be incomplete causing frequency-dependent attenuation and dispersion. The analysis of these effects requires a dynamic model of interaction of an elastic wave with an ensemble of fractures in a porous medium.

[4] A number of schemes tackling this dynamic problem in fractured porous rocks are currently available. Brajanovski et al. [2005] model a fractured medium as very thin, highly porous layers in a porous background. Their model implies that these fractures are of infinite extent and therefore is valid when fracture spacing is much smaller than fracture length (diameter). The case of finite-size fractures was considered by Hudson et al. [1996], who model fractures as thin pennyshaped voids, and account for fluid flow effects by applying the diffusion equation to a single crack and ignoring interaction between cracks. This approximation however leads to 


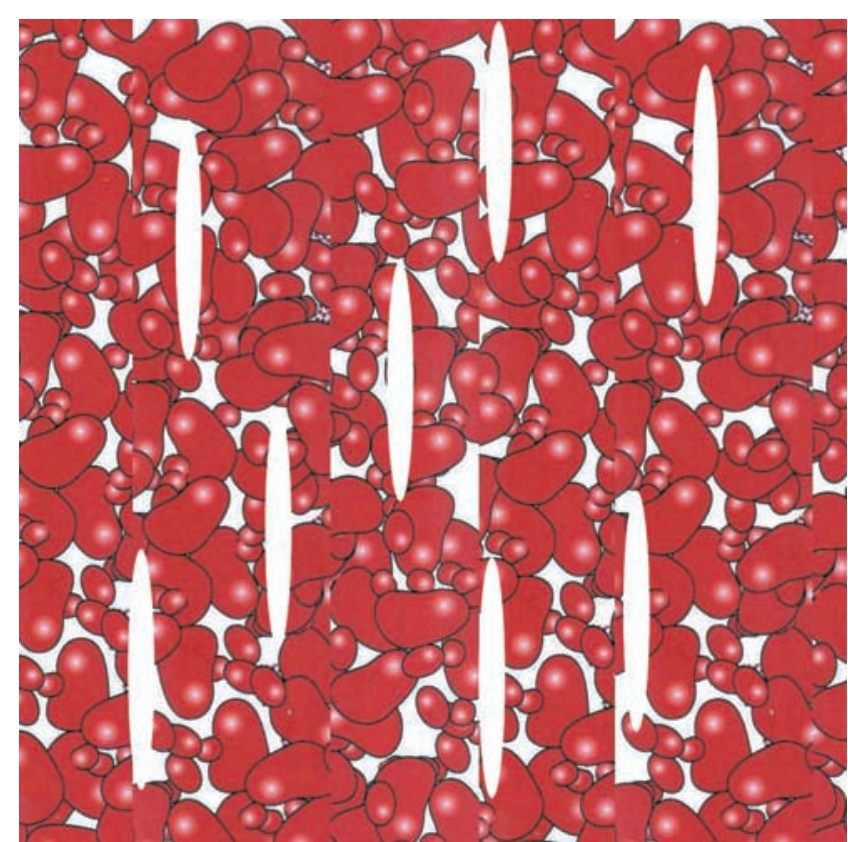

Figure 1. A porous medium containing a sparse distribution of cracks.

some unphysical effects, such as the result that the anisotropy of the fluid-saturated fractured and porous rock in the lowfrequency limit is the same as for the dry rock [Hudson et al., 2001; Chapman, 2003; Brown and Gurevich, 2004].

[5] Chapman [2003] and Maultzsch et al. [2003] analyze frequency-dependent anisotropy caused by the presence of mesoscale fractures in a porous rock, by considering connectivity of individual fractures, pores and microcracks. A more general computational model which can take account of pores and fractures of any size and shape was proposed by Jakobsen et al. [2003] using the T-matrix approximation, commonly used to study effective properties of heterogeneous media. In the T-matrix approximation the effect of voids (pores, fractures) is introduced as a perturbation of the solution for the elastic background medium.

[6] An alternative approach is to model the effect of fractures as a perturbation with respect to an isotropic porous background medium. This approach seems attractive because it allows us to use all the machinery of the theory of wave propagation in fluid-saturated porous media, known as the theory of poroelasticity [Biot, 1962], without specifying individual shapes of grains or pores. It also seems logical to assume that the perturbation of the porous medium caused by the introduction of fractures will be much smaller than the perturbation caused by putting all the pores and fractures into an elastic solid.

[7] In this paper we simulate the effect of fractures by considering them to be thin circular cracks in a poroelastic background. We assume that the cracks are mesoscopic (large compared to the pore size, but small compared to the fast wave wavelength). Using the solution of the scattering problem for a single crack [Galvin and Gurevich, 2007] and the multiple-scattering theory of Waterman and Truell [1961] we estimate the attenuation and dispersion of elastic waves taking place in a porous medium containing a sparse distribution of such cracks (Figure 1).

\section{Equations of Poroelasticity}

[8] We consider a system of aligned penny-shaped cracks in a fluid-saturated porous medium described by the equations of poroelasticity

$$
\begin{gathered}
\nabla \cdot \sigma=-\omega^{2}\left(\rho \mathbf{u}+\rho_{f} \mathbf{w}\right) \\
\nabla p=\omega^{2}\left(\rho_{f} \mathbf{u}+q \mathbf{w}\right)
\end{gathered}
$$

where $\mathbf{w}=\phi(\mathbf{U}-\mathbf{u})$ is the relative fluid displacement, $\phi$ is the medium porosity, $\mathbf{u}$ is the solid displacement, $\mathbf{U}$ is the average absolute fluid displacement, $\omega$ is the angular wave frequency, $\rho_{f}$ and $\rho$ are the densities of the fluid and of the overall medium. The parameter $q$ is a frequency-dependent coefficient responsible for viscous and inertial coupling between the solid and fluid displacements. Here and below we restrict ourselves to frequencies well below Biot's critical frequency, where fluid flow in the pores is of the Poiseuille type and $q(\omega) \approx i \eta / \kappa \omega[$ Biot, 1956] where $\eta$ is the fluid viscosity and $\kappa$ is the intrinsic permeability of the medium. $\sigma$ and $p$ are the total stress tensor and fluid pressure, which are related to the displacement vectors via the constitutive relations

$$
\begin{gathered}
\sigma=[(H-2 \mu) \nabla \cdot \mathbf{u}+\alpha M \nabla \cdot \mathbf{w}] \mathbf{I}+\mu\left[\nabla \mathbf{u}+(\nabla \mathbf{u})^{T}\right] \\
p=-\alpha M \nabla \cdot \mathbf{u}-M \nabla \cdot \mathbf{w} .
\end{gathered}
$$

In equations (3) and (4) $\mu$ is the shear modulus of the solid frame, $\alpha=1-K / K_{g}$ is the Biot-Willis coefficient [Biot and Willis, 1957],

$$
M=\left[\frac{(\alpha-\phi)}{K_{g}}+\frac{\phi}{K_{f}}\right]^{-1}
$$

is the so-called pore space modulus,

$$
H=K_{\text {sat }}+\frac{4}{3} \mu
$$

is the $\mathrm{P}$ wave modulus of the saturated poroelastic medium and $K_{s a t}$ is the bulk modulus of the saturated medium which is related to the bulk moduli of the fluid $K_{f}$, solid $K_{g}$, and dry skeleton $K$ by the Gassmann [1951] equation

$$
K_{\text {sat }}=K+\alpha^{2} M .
$$

[9] In the following we will consider the case of a single circular crack, which has axial symmetry. Thus there is no dependency upon the transverse angle $\theta$. Using equations (3) and (4) we can therefore decompose 
equations (1) and (2) into scalar equations in cylindrical coordinates $(r, \theta, z)$ :

$$
\begin{gathered}
\mu\left(\nabla^{2}-\frac{1}{r^{2}}\right) u_{r}+(\lambda+\mu) \frac{\partial e}{\partial r}+\rho \omega^{2} u_{r}-\alpha \frac{\partial p}{\partial r}=0 \\
\mu \nabla^{2} u_{z}+(\lambda+\mu) \frac{\partial e}{\partial z}+\rho \omega^{2} u_{z}-\alpha \frac{\partial p}{\partial z}=0 \\
\nabla^{2} p+\frac{i \omega b}{M} p+i \alpha b \omega e=0
\end{gathered}
$$

where $\lambda=K-4 \mu / 3, b=\eta / \kappa$, and $e$ is the cubical dilatation

$$
e=\nabla \cdot \mathbf{u}=\frac{1}{r} \frac{\partial}{\partial r}\left(r u_{r}\right)+\frac{\partial u_{z}}{\partial z}
$$

Equations (8)-(10) are supplemented by the constitutive relations

$$
\begin{gathered}
\sigma_{z z}=2 \mu \frac{\partial u_{z}}{\partial z}+\lambda e-\alpha p \\
\sigma_{r z}=\mu\left(\frac{\partial u_{r}}{\partial z}+\frac{\partial u_{z}}{\partial r}\right)
\end{gathered}
$$

and using equation (2) we have

$$
w_{z}=\frac{1}{i \omega b}\left(\frac{\partial p}{\partial z}-\rho_{f} \omega^{2} u_{z}\right)
$$

[10] The displacement and stress fields must obey the boundary conditions [Deresiewicz and Skalak, 1963] at the crack face, i.e. continuity of normal and tangential components of stresses, continuity of solid and relative fluid displacements and continuity of the pore fluid pressure. We assume that the circular cracks are in hydraulic communication with the surrounding porous medium. Here and below we assume that the fluid in the cracks (but not in the pores!) is incompressible. According to Hudson [1981], the compressibility of the crack-filling fluid can be neglected as long as

$$
\frac{K_{f}}{\mu} \gg \frac{c}{a}
$$

where as before $K_{f}$ is the bulk modulus of the fluid in the crack, $\mu$ is the shear modulus of the background medium, and $c / a$ is the aspect ratio of the crack. Note that in an elastic medium a crack filled with an incompressible fluid does not cause any scattering of a normally incident $\mathrm{P}$ wave. Thus in a porous medium such cracks will not cause any scattering of a normally incident $\mathrm{P}$ wave at frequencies where the size of the crack $a$ is comparable to the wavelength of the incident wave. However, there may still be scattering of incident energy due to fluid flow between the cracks and the pore space. As will be seen, this effect occurs at much lower frequencies where the crack radius is comparable to the diffusion length $1 /\left|k_{2}\right|$, where

$$
k_{2}=\left(\frac{i \omega \eta H}{\kappa M L}\right)^{1 / 2}
$$

is the complex wave number of Biot's slow wave (here $L=$ $\lambda+2 \mu=K+4 \mu / 3$ is the $\mathrm{P}$ wave modulus of the dry frame).

[11] In the next section we review the problem of scattering of an elastic wave in a porous medium by a single crack, and then present analysis of the scattering by an ensemble of cracks.

\section{Scattering by a Single Crack}

[12] Interaction of a plane elastic wave with a single crack in the porous medium described by the equations outlined above was considered by Galvin and Gurevich [2007]. An incident plane longitudinal wave, harmonic in time, propagates in the positive direction of the $z$ axis (normal to the crack plane $z=0$ ) of a cylindrical coordinate system.

[13] Since we have geometrical symmetry about the crack plane $z=0$, both the scattered and total displacement fields satisfy the equations of dynamic poroelasticity given above in the semi-infinite poroelastic medium $z \geq 0$. The incident wave can be represented as the displacement field $u_{z}^{(i)}=u_{0} e^{i k_{1} z}$, where $k_{1}$ is the wave number. The aim is to derive the scattered field $\mathbf{u}(\mathbf{r})$ that results from interaction between the incident wave and the crack, which occupies the circle $0 \leq r \leq a$ in the plane $z=0$. The total displacement field is therefore $\mathbf{u}^{(t)}(\mathbf{r})=u_{z}^{(i)} \mathbf{a}_{z}+\mathbf{u}(\mathbf{r})$, where $\mathbf{a}_{z}$ is a unit vector directed along the $z$ axis.

[14] For an incompressible fluid in the cracks, the volume fraction average of the normal displacement $(1-\phi) u_{z}+$ $\phi U_{z}=u_{z}+w_{z}$ through the face of each crack must be equal to zero. Continuity of total normal stress tells us that both $\sigma_{z z}^{(t)}$ and $p^{(t)}$ in the total field in the porous medium in the vicinity of the crack face must be equal to the pressure of the crack-filling fluid, $p^{(c)}$, so that $\sigma_{z z}^{(t)}=p^{(t)}$ or $\sigma_{z z}-p=-\left(\sigma_{z z}^{(i)}-p^{(i)}\right)$. Also, analogously to the elastic case, $\sigma_{r z}$ is everywhere zero, and $u_{z}=w_{z}=0$ due to symmetry considerations. Expressing stress and fluid pressure in terms of displacement via constitutive relations (12) and (4) our boundary conditions can be written:

$$
\begin{gathered}
\sigma_{r z}=0 \quad 0 \leq r<\infty \\
u_{z}=0 \quad a<r<\infty \\
w_{z}=0 \quad a<r<\infty \\
u_{z}+w_{z}=0 \quad 0 \leq r \leq a \\
\sigma_{z z}+p=-i k_{1}(H-\alpha M) u_{0} \quad 0 \leq r \leq a
\end{gathered}
$$


where it is noted that conditions (18)-(20) can be combined to give the single condition

$$
u_{z}+w_{z}=0 \quad 0 \leq r \leq \infty .
$$

[15] Galvin and Gurevich [2007] obtained a general solution to equations (8)-(10) using Hankel transform techniques, reducing the scattering problem to a pair of dual integral equations:

$$
\begin{gathered}
\int_{0}^{\infty} y[1+T(y)] B(y) J_{0}(y r) d y=-p_{0} \quad 0 \leq r \leq a, \\
\int_{0}^{\infty} B(y) J_{0}(y r) d y=0 \quad a<r<\infty
\end{gathered}
$$

where

$$
\begin{gathered}
T(y)=\left[1+\frac{\alpha M k_{3}^{2}}{H\left(2 y^{2}-k_{3}^{2}\right)}\right]\left[T_{1}(y)-T_{2}(y)\right]-1, \\
T_{1}(y)=\frac{M\left(k_{2}^{2} L-2 \alpha \mu y^{2}\right)\left[4 \alpha g y^{2}\left(y^{2}-q_{2} q_{3}\right)-k_{2}^{2}\left(2 y^{2}-k_{3}^{2}\right)\right]}{2 \mu H(1-g) k_{2}^{2} q_{2} y\left[2 y^{2}-k_{3}^{2}\left(1-\frac{\alpha M}{H}\right)\right]} \\
T_{2}(y)=\frac{\alpha g\left[\left(2 y^{2}-k_{3}^{2}\right)^{2}-4 y^{2} q_{1} q_{3}\right]+\left(2 y^{2}-k_{3}^{2}\right)\left(k_{0}^{2}-k_{1}^{2}\right)}{2 \alpha g(1-g) k_{3}^{2} q_{1} y},
\end{gathered}
$$

$k_{3}$ is the shearwave wave number, $q_{i}=\sqrt{y^{2}-k_{i}^{2}}$ (so that $i q_{i}=\sqrt{k_{i}^{2}-y^{2}}$ is the axial wave number), $g=\mu / L$, and $p_{0}=i k_{1}(H-\alpha M) u_{0}$ is the incident pressure. The function $B(y)$ is defined by

$$
B(y)=-\frac{2 \alpha \mu(1-g) k_{3}^{2} q_{1} y}{L\left[2 y^{2}-k_{3}^{2}\left(1-\frac{\alpha M}{H}\right)\right]} A_{1}(y),
$$

where $A_{1}(y)$ is the spectral amplitude of the scattered fast compressional wave.

[16] Dual integral equations (23)-(24) were then reduced to a single Fredholm equation of the second kind:

$$
B(x)+\frac{1}{\pi} \int_{0}^{\infty} R(x, y) T(y) B(y) d y=-p_{0} S(x),
$$

where

$$
\begin{gathered}
R(x, y)=\frac{\sin a(x-y)}{x-y}-\frac{\sin a(x+y)}{x+y}, \\
S(x)=\frac{2}{\pi} \frac{\sin a x-a x \cos a x}{x^{2}},
\end{gathered}
$$

and $x$ and $y$ are radial wave numbers. Since an analytical solution to equation (29) exists only when its kernal function
$R(x, y) T(y)$ is separable, in general one must obtain $B(x)$ via numerical methods.

\section{A Sparse Distribution of Cracks}

[17] The multiple-scattering theorem of Waterman and Truell [1961] provides a method to compute attenuation and dispersion of seismic waves in a medium with randomly distributed inhomogeneities, if the far-field amplitude of the scattering by a single inhomogeneity is known. According to Waterman and Truell [1961], effective wave number may be calculated from the amplitudes of the scattered field as

$$
\left(\frac{k^{*}}{k_{1}}\right)^{2}=\left[1+\frac{2 \pi n_{0} f(0)}{k_{1}^{2}}\right]^{2}-\left[\frac{2 \pi n_{0} f(\pi)}{k_{1}^{2}}\right]^{2}
$$

where $k_{1}$ is the real wave number of the fast $\mathrm{P}$ wave, $n_{0}$ is the density or number of scatterers per unit volume and $f(0)$, $f(\pi)$ are far-field amplitudes of the fast $\mathrm{P}$ wave scattered in the forward and backward direction (with respect to the incident wave) by a single inclusion. For a sufficiently small concentration of inclusions quadratic terms in equation (32) can be neglected, which yields

$$
k^{*}=k_{1}\left[1+\frac{4 \pi n_{0} f(0)}{k_{1}^{2}}\right]^{1 / 2} \approx k_{1}\left[1+\frac{2 \pi n_{0} f(0)}{k_{1}^{2}}\right]
$$

The real part of equation (33) gives the effective velocity $v^{*}$ in media with a low concentration of scatterers

$$
\frac{1}{v^{*}}=\frac{1}{v_{1}}\left[1+\frac{2 \pi n_{0}}{k_{1}^{2}} \operatorname{Re}\{f(0)\}\right]
$$

The imaginary part of equation (33) gives the dimensionless attenuation (inverse quality factor)

$$
Q^{-1}=\frac{4 \pi n_{0}}{k_{1}^{2}} \operatorname{Im}\{f(0)\}
$$

The above expressions (34) and (35) allow us to model the dispersion and attenuation due to the scattering of a plane elastic wave by poroelastic inclusions randomly distributed throughout a poroelastic medium. In this study we investigate the special case of dispersion and attenuation due to aligned cracks using the solution for the scattering by a circular crack given in the previous section. Our $f(0)$ is obtained analogously to the elastic case [Robertson, 1967] by considering the far-field asymptotics of the axial displacement

$$
u_{z}=\int_{0}^{\infty}\left[A_{3}(y) e^{-q_{3} z}-\frac{\alpha}{L} \sum_{i=1}^{2} A_{i}(y) q_{i} e^{-q_{i}}\right] y J_{0}(y r) d y
$$

[Galvin and Gurevich, 2007] where the $A_{i}(y)$ are the spectral amplitudes of the fast, slow and shear waves scattered by a single crack. This yields (see Appendix A)

$$
f(0)=-\frac{i k_{1}(H-\alpha M)}{2 \mu H(1-g)} \lim _{y \rightarrow 0} \frac{B(y)}{y} .
$$




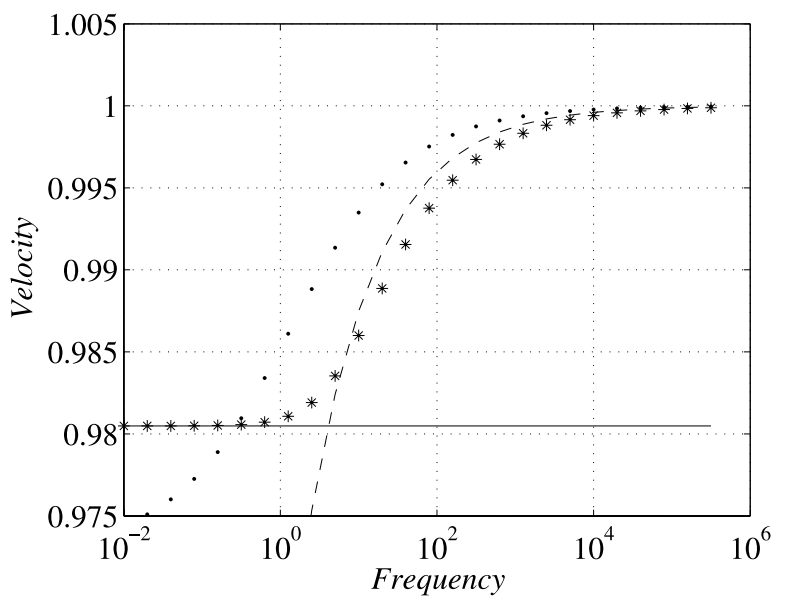

Figure 2. Dimensionless velocity as a function of dimensionless frequency: numerical solution (asterisks), low-frequency asymptotic (solid line), high-frequency asymptotic (dashed line), and the Hudson et al. [1996] equant porosity model (dots).

Substitution of $f(0)$ given by equation (37) into equations (34) and (35) gives the frequency dependency of the fast wave velocity and attenuation in terms of the solution $B(x)$ of the integral equation (29).

[18] The Fredholm equation (29) needs to be solved for every frequency (which is a parameter of the kernel function $R(x, y) T(y)$ and the right-hand side $S(x))$. This equation does not have a general analytical solution for arbitrary frequency, and has to be solved numerically. However, the equation can be greatly simplified in the limiting cases of low and high frequencies, resulting in asymptotic analytical solutions. Numerical and asymptotic analytical solutions are presented in the next sections.

\section{Numerical Solution}

[19] The numerical solution of the Fredholm equation (29) can be easily obtained by the method of quadratures. Figures 2 and 3 show this solution in terms of effective velocity $v(\omega)=\omega / \operatorname{Re} k^{*}$ (normalized by crack-free saturated velocity $v_{1}$ ) and dimensionless attenuation $Q^{-1}=2 \mathrm{Re} k^{*} / \operatorname{Im} k^{*}$ as functions of dimensionless frequency $\omega^{\prime}=\left|k_{2} a\right|^{2}$. Also shown in Figure 3 are asymptotic solutions in the low- and high-frequency limits (explained in the following sections), the equant porosity model of Hudson et al. [1996], and the result for spherical inclusions [Ciz et al., 2006] for comparison. The solution exhibits a typical relaxation peak around a normalized frequency $\omega^{\prime}$ of about 10, or at circular frequency $f=\omega / 2 \pi \simeq 2 \kappa M(K+4 \mu / 3) / H \eta a^{2}$, the frequency where the fluid diffusion length $1 /\left|k_{2}\right|$ is of the order of the crack radius $a$.

\section{Analytical Solution}

\subsection{Mesoscopic Cracks}

[20] The kernel function $T(y)$ as given by equation (25) is general with regards to the size of the crack relative to the incident wavelength. However, we are mainly interested in situations where the scattering is due to wave-induced fluid flow to and from the cracks (i.e. the slow wave). For the scattering to be predominantly due to fluid flow, the crack size must be small relative to the wavelength of the incident compressional wave, $k_{1} a \ll 1$ (mesoscopic cracks), so that scattering into the slow wave mode is dominant. For significant slow wave scattering to take place the radial wave number $y$ should be of the order of $1 / a$, or in other words $y \gg k_{1}$ and we can approximate expression (25) as

$$
T(y) \approx M \frac{\left(2 \alpha g y^{2}-k_{2}^{2}\right)^{2}-2 y q_{2} \alpha g\left[k_{2}^{2}(\alpha g-2)+2 \alpha y^{2} g\right]}{2 H g(g-1) y q_{2} k_{2}^{2}} .
$$

We now obtain asymptotic solutions in the low- and highfrequency limits. Note that we are still in the low-frequency regime of Biot theory, the low- and high-frequency limits we evaluate here are with respect to the extent to which fluid is able to diffuse between crack and pore space during passage of the wavefield. That is, in the low-frequency limit there is plenty of time available for fluid flow to occur, the diffusion length can be taken large compared with the crack radius, or $\left|k_{2}\right| a \ll 1$. At increasingly high frequencies there is no time available for appreciable fluid flow to occur and therefore the diffusion length can be taken as small compared with the crack radius, $\left|k_{2}\right| a \gg 1$.

\subsection{Low-Frequency Asymptote}

[21] For low frequencies $\left|k_{2}\right| a \ll 1$. Then $S(y)$ only contributes significantly to the integral in equation (29) for $y$ of the order $1 / a$. For $y \ll 1 / a, S(y)$ is small. Thus $T(y)$ only contributes to the integral in equation (29) for $y \gg\left|k_{2}\right|$. In this case equation (38) can be simplified to give

$$
T_{\text {low }}(y) \approx \frac{-M k_{2}^{2}\left(2 L^{2}+3 \alpha^{2} \mu^{2}-4 \alpha \mu L\right)}{4 H \mu(L-\mu) y^{2}} .
$$

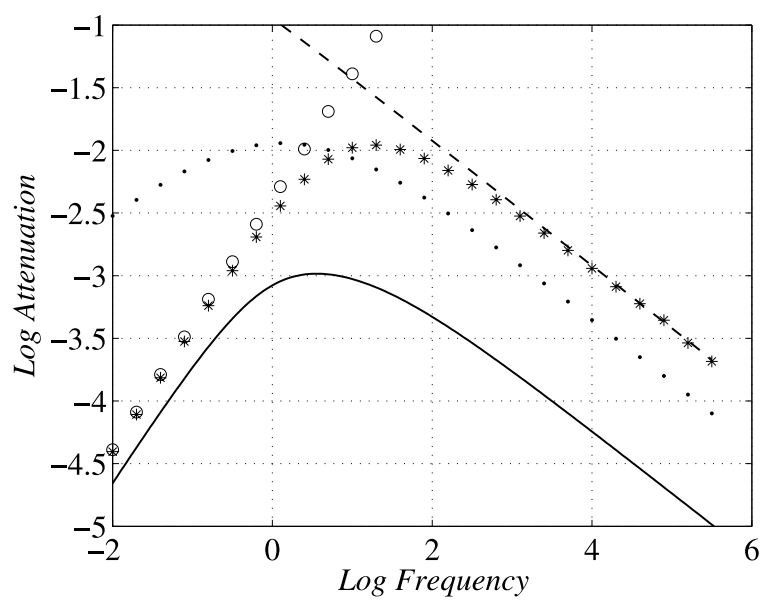

Figure 3. Dimensionless attenuation as a function of dimensionless frequency: numerical solution (asterisks), low-frequency asymptotic (circles), high-frequency asymptotic (dashed line), the Hudson et al. [1996] equant porosity model (dots), and the result for spherical inclusions (solid line). Crack and sphere radius are equal. 
At low frequencies the contribution of the integral in equation (29) is small relative to the RHS and therefore equation (29) can be solved by iteration. That is, assuming an initial solution $B(x)=-p_{0} S(x)$ and then substituting $B(y)=$ $-p_{0} S(y)$ and $T(y) \approx T_{\text {low }}(y)$ into equation (29), yields

$$
B(x)=-p_{0} S(x)+\frac{p_{0}}{\pi} \int_{0}^{\infty} R(x, y) T(y) S(y) d y .
$$

Because we actually require the limit of $B(x) / x$ as $x \rightarrow 0$ we can simplify the integral in equation (40) by rewriting it

$$
\lim _{x \rightarrow 0} \frac{B(x)}{x}=-p_{0} \lim _{x \rightarrow 0} \frac{S(x)}{x}+\frac{p_{0}}{\pi} \int_{0}^{\infty} \lim _{x \rightarrow 0} \frac{R(x, y)}{x} T(y) S(y) d y
$$

and then evaluating the limits on the RHS. Expanding $R(x$, $y) / x$ using the trigonometric identity

$$
\sin (A \pm B)=\sin A \cos B \pm \cos A \sin B
$$

we obtain

$$
\frac{R(x, y)}{x}=\frac{2(y \sin a x \cos a y-x \cos a x \sin a y)}{x\left(x^{2}-y^{2}\right)} .
$$

As $x \rightarrow 0, \sin a x \approx a x$ and $\cos a x \approx 1$ so that equation (43) simplifies to

$$
\frac{R(x, y)}{x} \approx \frac{2(\sin a y-a y \cos a y)}{y^{2}}=\pi S(y) .
$$

At small $x$,

$$
\sin a x-a x \cos a x \approx \frac{(a x)^{3}}{3}
$$

and hence

$$
\frac{S(x)}{x} \approx \frac{2 a^{3}}{3 \pi}
$$

Therefore equation (41) simplifies to

$$
\lim _{x \rightarrow 0} \frac{B(x)}{x}=-\frac{2 p_{0} a^{3}}{3 \pi}+p_{0} \int_{0}^{\infty} T_{l o w}(y)[S(y)]^{2} d y
$$

which, upon evaluation of the integral, yields

$$
\lim _{x \rightarrow 0} \frac{B(x)}{x}=\frac{p_{0} a^{3}\left[M\left(k_{2} a\right)^{2}\left(4 \alpha \mu L-2 L^{2}-3 \alpha^{2} \mu^{2}\right)-10 H \mu(L-\mu)\right]}{15 \pi H \mu(L-\mu)} .
$$

Thus equation (37) yields

$$
f_{\text {low }}(0)=\frac{\left[5+\frac{M\left(2-4 \alpha g+3 \alpha^{2} g^{2}\right)\left(k_{2} a\right)^{2}}{2 H g(1-g)}\right](H-\alpha M)^{2} k_{1}^{2} a^{3}}{15 \pi \mu H(1-g)} .
$$

By substituting equation (49) into (34) and taking the real part, one can obtain an expression for effective velocity in the low-frequency limit

$$
v^{*}=v_{1}\left[1-\frac{2 \varepsilon(H-\alpha M)^{2}}{3 \mu H(1-g)}\right] \text {. }
$$

In equation $(50) v_{1}=\omega / k_{1}=(H / \rho)^{1 / 2}$ is the velocity of the fast compressional wave in the porous host (crack-free fluidsaturated porous medium) and $\varepsilon=n_{0} a^{3}=(3 / 4 \pi)(a / b) \phi_{c}$ is the crack density parameter [Hudson, 1980] where $\phi_{c}=$ $(4 / 3) \pi a^{2} b n_{0}$ is the additional porosity present due to the cracks.

[22] Low-frequency attenuation $Q^{-1}$ is defined by the imaginary part of the function $f_{\text {low }}(0)$,

$$
Q_{l o w}^{-1}=\frac{2 M(H-\alpha M)^{2}\left(2-4 \alpha g+3 \alpha^{2} g^{2}\right)\left|k_{2} a\right|^{2} \varepsilon}{15 \mu H^{2} g(1-g)^{2}},
$$

and is proportional to $\left|k_{2} a\right|^{2}$, that is, to the first power of frequency.

\subsection{High-Frequency Asymptote}

[23] For high frequencies $\left|k_{2}\right| a \gg 1$. To analyze this case we note that function $R(x, y)$ as given by equation $(30)$ is a function oscillating with a period of $2 \pi / a$ and decaying with $y$ as $y^{-1}$. At the same time, $T$ is also a function decaying with a positive power of $y$. Thus most of the contribution to the integral in equation (29) comes from the interval $0<y<$ $y_{0}$, that is,

$$
B(x)+\frac{1}{\pi} \int_{0}^{y_{0}} R(x, y) T(y) B(y) d y \approx-p_{0} S(x),
$$

where $y_{0}$ is on the order of $1 / a$. For high frequencies $\left|k_{2}\right| a \gg$ 1 and thus $y_{0} \ll\left|k_{2}\right|$. Hence we can simplify expression (38) assuming that $y \ll\left|k_{2}\right|$,

$$
T_{\text {high }}(y) \approx \frac{-i M L^{2} k_{2}}{2 H \mu(L-\mu) y} .
$$

In this limit we can obtain an analytical solution directly from dual equations (23) and (24). Since $T_{h i g h}(y)$ is much larger than 1 in equation (23), substituting $T_{\text {high }}(y)$ yields a single integral equation for $B(y)$ :

$$
\int_{0}^{\infty} B(y) J_{0}(y r) d y=D(r)
$$

where

$$
D(r)=\begin{array}{cc}
\frac{-2 i g H(1-g) p_{0}}{M k_{2}} & 0 \leq r \leq a \\
0 & a<r \leq \infty .
\end{array}
$$

Note that the left hand side of equation (54) is simply the Hankel transform of the function $B(y) / y$. Thus $B(y) / y$ can be 
obtained as the inverse Hankel transform of the right hand side,

$$
\frac{B(y)}{y}=\int_{0}^{\infty} D(r) J_{0}(y r) r d r=\frac{-2 i g H(1-g) p_{0}}{M k_{2}} \int_{0}^{a} J_{0}(y r) r d r .
$$

For equation (37) we only need the limit of $B(y) / y$ for small $y$. Noting that $J_{0}(y r) \rightarrow 1$ for small arguments, we obtain

$$
\lim _{y \rightarrow 0} \frac{B(y)}{y}=\frac{-i g H(1-g) p_{0} a^{2}}{M k_{2}} .
$$

Substitution of this result into equation (37) yields:

$$
f_{\text {high }}(0)=-\frac{i\left(k_{1} a\right)^{2}(H-\alpha M)^{2}}{2 M L k_{2}} .
$$

By substituting this expression into the dispersion equation (34) one can see that its relative contribution to the real part of the effective wave number vanishes in the high-frequency limit, implying that the velocity in that limit tends to the velocity in the porous crack-free medium. This result is logical as at sufficiently high frequencies the fluid has no time to move between pores and cracks, and therefore the cracks behave as if they were isolated. In particular, the dry case is excluded, except in the static limit.

[24] Attenuation at high frequencies reads

$$
Q_{\text {high }}^{-1}=\frac{\sqrt{2} \pi \varepsilon(H-\alpha M)^{2}}{M L\left|k_{2} a\right|},
$$

and thus scales with $\omega^{-1 / 2}$.

\subsection{Peak Frequency}

[25] Finally, the asymptotic results of this section allow us to obtain an expression for the characteristic frequency of the dissipation mechanism that exists due to wave-induced flow between pores and cracks. Earlier we argued from physical principles that characteristic frequency $\omega_{c}$ of this mechanism is such that the diffusion length $1 /\left|k_{2}\right|$ is of the order of the crack size $a$,

$$
\left(\frac{i \omega_{c} \eta H}{\kappa M L}\right)^{-1 / 2} \sim a
$$

or

$$
\omega_{c} \sim \frac{\kappa}{\eta a^{2}} \frac{M L}{H} .
$$

In the stiff frame approximation $K_{f} \ll K<K_{g}$ we have $H \approx$ $L, M \approx K_{f} / \phi$ and expression (61) simplifies to

$$
\omega_{c} \sim \frac{\kappa}{\eta a^{2}} \frac{K_{f}}{\phi} .
$$

[26] A more precise estimate of the characteristic frequency can be obtained by considering the frequency at which the low- and high-frequency asymptotic solutions (51) and (59) are equal (intersection of the asymptotes):

$$
\frac{2 M(H-\alpha M)^{2}\left(2-4 \alpha g+3 \alpha^{2} g^{2}\right)\left|k_{2} a\right|^{2} \varepsilon}{15 \mu H^{2} g(1-g)^{2}}=\frac{\sqrt{2} \pi \varepsilon(H-\alpha M)^{2} g}{\mu M\left|k_{2} a\right|}
$$

or

$$
\left|k_{2} a\right|^{3}=\frac{15 \pi \sqrt{2} H^{2} g^{2}(1-g)^{2}}{2 M^{2}\left(2-4 \alpha g+3 \alpha^{2} g^{2}\right)} .
$$

Which, using equation (16) yields the following expression for characteristic frequency:

$$
\omega_{c}=\frac{\kappa M L}{\eta a^{2} H}\left[\frac{15 \pi \sqrt{2} H^{2} g^{2}(1-g)^{2}}{2 M^{2}\left(2-4 \alpha g+3 \alpha^{2} g^{2}\right)}\right]^{2 / 3} .
$$

One can see that this precise estimate of the peak frequency has the same dependence on permeability, viscosity and crack size as the intuitive estimate (62), but differs in its dependency on porosity and both fluid and solid moduli.

[27] Finally, we can estimate the peak value of attenuation by substituting the expression for $\omega_{c}$ (or $k_{2} a$ ) into one of the asymptotic solutions. This gives

$$
Q_{\max }^{-1}=\frac{\varepsilon(H-\alpha M)^{2}}{\mu}\left[\frac{4 \pi^{2} g\left(2-4 \alpha g+3 \alpha^{2} g^{2}\right)}{15 M H^{2}(1-g)^{2}}\right]^{1 / 3}
$$

or, for the common stiff case $\alpha M \ll L, K_{f} \ll K_{g}$,

$$
Q_{\max }^{-1}=\left[\frac{4 \pi^{2}\left(2-4 \alpha g+3 \alpha^{2} g^{2}\right) L \phi}{15 g^{2}(1-g)^{2} K_{f}}\right]^{1 / 3} \varepsilon
$$

Apart from the obvious fact that maximum attenuation is proportional to crack density, this shows a counterintuitive result that peak attenuation increases with decreasing fluid bulk modulus (albeit rather weakly). However, one should remember that the theory presented here is only valid for relatively stiff fluids obeying the condition (15) and not for gas-saturated or dry media.

\section{Discussion}

\subsection{Gassmann Consistency}

[28] In the low-frequency limit the pressure throughout the pore space and fractures should be equilibrated. In his seminal paper Gassmann [1951] showed that in this case the elastic properties of the saturated medium are uniquely defined by the elastic properties of the dry frame, porosity, and the bulk moduli of the solid grain material and the saturating fluid. For the isotropic case Gassmann derived an expression for the undrained bulk modulus of the saturated medium, which is widely used for fluid substitution in porous rocks. However, Gassmann [1951] also derived a more general equation for the case where the frame is macroscopically anisotropic (but is still made up of a single isotropic solid material). This equation was later generalized 
by Brown and Korringa [1975] to materials made of anisotropic and microheterogeneous solid material.

[29] A porous medium with aligned fractures is macroscopically anisotropic (transversely isotropic) and is made of one isotropic solid. Therefore it should be consistent with the anisotropic Gassmann [1951] equation. This particular case was analyzed by Gurevich [2003] who derived explicit expressions for the stiffness tensor of the saturated medium as a function of porosity and elastic moduli of the background medium, solid and fluid bulk moduli, and fracture weakness (which for sparsely populated cracks can be uniquely related to fracture density).

[30] The results of Gurevich [2003] are exact expressions valid for any fracture weakness. In order to compare our low-frequency result with the expression of Gurevich [2003], we expand that expression in powers of fracture density and retain only the linear term. The resulting expression for the plane deformation stiffness $c_{33}^{\text {sat }}$ along the symmetry axis $z$ is

$$
c_{33}^{\text {sat }}=H\left[1-\frac{4 \varepsilon(H-\alpha M)^{2}}{3 \mu H(1-g)}\right] .
$$

[31] For the velocity of compressional waves propagating along the $z$ axis equation (68) gives an equation identical to equation (50) (again, for small crack density). This confirms that our result at low frequencies is asymptotically (e.g., in sparse limit) consistent with the Gassmann's theory. This Gassmann consistency is an important feature of the model presented here and shows that the hydraulic interaction between cracks and pores is properly accounted for.

[32] The dry case $K_{f}=M=0$ violates the condition (15) making the present theory invalid. However, if we formally take the limit $M \rightarrow 0$, then equation (68) gives the following expression for the dry axial $\mathrm{P}$ wave modulus

$$
c_{33}^{d r y}=H\left[1-\frac{4 \varepsilon}{3 g(1-g)}\right]
$$

or, for dry $\mathrm{P}$ wave velocity

$$
v^{d r y}=v_{1}\left[1-\frac{2 \varepsilon}{3 g(1-g)}\right] \text {. }
$$

This coincides with the well-known expression for the velocity of compressional waves propagating perpendicular to a system of dry open cracks in an elastic medium in the limit of low crack density [Hudson, 1980]. The lowfrequency velocity gives the correct result in the dry limit (which violates the condition (15)) because at low frequencies fluid in the cracks and pores is in full pressure equilibrium, and thus the stiffness of the fluid in the cracks is irrelevant.

\subsection{Comparison With the Equant Porosity Model of Hudson et al. [1996]}

[33] Hudson et al. [1996] developed a model of a cracked medium with equant porosity, where pressure within a crack is relieved into the host porous material by diffusion; see also Hudson et al. [2001]. We can compare the predictions of our model with the equant porosity model (EPM) of Hudson et al. [1996] as the two models differ only in the assumptions regarding fluid flow, and all the parameters have the same physical meaning. The effective velocity for wave propagation in the EPM is

$$
v_{H}^{*}=v_{1 H}\left[1-\frac{4 \varepsilon L^{2}}{3 \mu\left(1+K_{H}\right)(L-\mu)}\right] \text {, }
$$

where for thin cracks (thickness less than the fluid diffusion length)

$$
K_{H} \approx\left(\frac{1+i}{3}\right) \frac{L}{\mu}\left(\frac{K_{f}}{L-\mu}\right) \frac{a}{J}
$$

and

$$
J^{2}=\frac{\phi K_{f} \kappa}{2 \omega \eta}
$$

Figure 2 shows the dispersion predicted by both models as well as high and low-frequency asymptotes for our model. At high frequencies both models predict no deviation from the background velocity. This is logical as in the highfrequency limit of both models there is no time available for appreciable fluid flow to occur, and hence there will be no flow-induced dispersion. At low frequencies our model is consistent with the static Gassmann theory, whereas the EPM is not. The possible reason for this disagreement is that hydraulic interaction between different cracks is not accounted for in the EPM.

[34] Figure 3 shows the attenuation curves for both models, and the low and high frequency asymptotes for our model. The attenuation asymptotes for the EPM are

$$
Q_{\text {lowH }}^{-1}=\frac{8 \sqrt{2} \varepsilon a}{9 \mu g(1-g)^{2}} \sqrt{\frac{K_{f} \eta \omega}{\phi \kappa}}
$$

for low frequencies and

$$
Q_{h i g h H}^{-1}=\frac{2 \sqrt{2} \varepsilon L}{K_{f} a} \sqrt{\frac{\phi K_{f} \kappa}{\eta \omega}}
$$

for high frequencies. We can more closely compare our asymptotes (51) and (59) with the EPM by taking a rigidframe approximation, which yields

$$
Q_{\text {low }}^{-1} \approx \frac{4\left(2-4 \alpha g+3 \alpha^{2} g^{2}\right) a^{2} \varepsilon \eta \omega}{15 \mu g(1-g)^{2} \kappa}
$$

for low frequencies and

$$
Q_{\text {high }}^{-1} \approx \frac{\pi \varepsilon L}{K_{f} a} \sqrt{\frac{\phi K_{f} \kappa}{2 \eta \omega}}
$$

for high frequencies. At high frequencies the behavior of the two models is almost identical. At low frequencies the predictions are significantly different, as our model predicts 
that $Q^{-1}$ will be proportional to $\omega$ whereas the EPM predicts $Q^{-1}$ being proportional to $\sqrt{\omega}$. The attenuation curves of Figure 3 also show that both models predict an attenuation peak of the same magnitude, although the EPM predicts the peak occurring at a lower frequency.

[35] An approximation for the nature of the characteristic attenuation peaks shown in Figure 3 can be obtained by comparing the expressions for characteristic frequencies. The characteristic frequency for the EPM can be obtained by equating the low- and high-frequency asymptotes of attenuation,

$$
\omega_{E P M}=\frac{\kappa}{\eta a^{2}} \frac{9 \phi \mu^{2}(1-g)^{2}}{4 K_{f}}=\frac{\kappa}{\eta a^{2}} \frac{9 \phi g^{2}(\lambda+\mu)^{2}}{4 K_{f}} .
$$

Comparing this expression with the corresponding expression (65) for $\omega_{c}$ from our theory, we see that these expressions show similar dependencies on permeability, fluid viscosity and crack radius, but are somewhat different with regards to fluid and matrix moduli, and porosity.

\subsection{Comparison With the Model of Chapman [2003]}

[36] It is also interesting to compare our results with the model of Chapman [2003]. Chapman considers coupled fluid motion between cracks and equant background porosity on two scales: a grain scale and a much larger mesoscopic fracture scale. Each fracture is assumed to be hydraulically connected to a fixed number of spherical pores.

[37] As far as velocities are concerned, Chapman's [2003] model predicts the same low- and high-frequency limiting behavior as our model: Gassmann velocity in the low-frequency limit and velocity of a crack-free medium in the high frequency limit. Furthermore, both models predict the same frequency scaling of attenuation at low frequencies. At high frequencies, however, Chapman's model predicts that $Q^{-1}$ will scale with $\omega^{-1}$ whereas our model predicts a scaling with $\omega^{-1 / 2}$. The characteristic attenuation peak frequencies are in agreement as long as it is assumed that Chapman's relaxation time $\tau_{f}$ (inverse of characteristic frequency) is proportional to $(a / d)^{2}$ where $a$ is the fracture size and $d$ is the grain size, and not to $(a / d)$, as in the work of Maultzsch et al. [2003]. The nature of these differences is as yet unclear and is the subject of an ongoing analysis. We shall note however that the scaling of attenuation with $\omega^{-1 / 2}$ at high frequencies is consistent with the EPM model discussed above, and is a common effect of heterogeneities in poroelastic media when the characteristic size of the inhomogeneities is much larger than the diffusion length $1 /\left|k_{2}\right|$ [White, 1975; Norris, 1993; Gurevich and Lopatnikov, 1995; Johnson, 2001; Müller and Gurevich, 2005].

\subsection{Comparison With Planar Fractures}

[38] A theory of attenuation and dispersion of elastic waves in a poroelastic medium permeated by a system of parallel planar fractures was recently proposed by Brajanovski et al. [2005], who modeled fractures as thin periodically spaced flat layers with very high porosity $\phi_{f} \rightarrow 1$. The theory of Brajanovski et al. [2005] gives the same scaling laws for attenuation at low $\left(Q^{-1} \propto \omega\right)$ and high $\left(Q^{-1} \propto \omega^{-1 / 2}\right)$ frequencies as the present theory. However, direct quantitative comparison of the two theories is not possible since the ensembles of planar and finite circular cracks are controlled by different parameters. It is nevertheless instructive to compare the characteristic frequencies of the two theories. The theory of planar fractures has two characteristic frequencies [Brajanovski et al., 2006] but only one of them is relevant for small crack densities [Lambert et al., 2006]. The expression for this frequency is [Brajanovski et al., 2006], equation (14)

$$
\omega_{P}=2 \sqrt[3]{36} \Delta_{N}^{-4 / 3} \frac{\kappa L}{h^{2} \eta}\left(\frac{H}{M}\right)^{1 / 3}
$$

where $h$ is characteristic fracture spacing and $\Delta_{N}$ is the socalled relative fracture weakness. In order to compare this expression to our expression for $\omega_{c}$ we need to relate $\Delta_{N}$ to the crack density. For low crack density we have [Schoenberg and Douma, 1988]

$$
\Delta_{N}=\frac{4 \varepsilon L^{2}}{3 \mu(L-\mu)}=\frac{4 \varepsilon}{3 g(1-g)}
$$

and hence

$$
\omega_{P}=\frac{9}{2} \frac{\kappa}{h^{2} \eta} \mu(1-g)\left[g(1-g) \frac{H}{M}\right]^{1 / 3} \varepsilon^{-4 / 3} .
$$

The striking difference between $\omega_{P}$ and any other characteristic frequencies mentioned earlier is its strong dependence on fracture weakness or crack density. However, direct comparison of these characteristic frequencies is not entirely applicable, because for sparse finite-size circular cracks their effect on elastic properties of, say, dry material is entirely controlled by their geometrical configuration, that is, by crack radius and number density. For planar fractures this is not the case: because normal fracture weakness $\Delta_{N}$ and fracture spacing $h$ are completely independent parameters. Nevertheless, it is interesting to note that $\omega_{P}$ is proportional to $\kappa / h^{2} \eta$. Thus the dependency of $\omega_{P}$ on permeability and viscosity is the same as in the present theory, while the crack size in the expression for $\omega_{c}$ is replaced by fracture spacing. This is the result of the fact that in the theory of parallel planar fractures the waves scattered by individual fractures interfere coherently, with interference naturally controlled by the distance between fractures. Also of note is the fact that the dependencies of $\omega_{c}$, equation (65), and $\omega_{P}$, equation (81), on $H$ and $M$ (and thus on the porosity and elastic moduli of the matrix and fluid) also show some similarities. Furthermore, a more detailed comparison shows that the high-frequency attenuation asymptotes for the two models are exactly the same for the same specific surface of fractures [Gurevich et al., 2007]. This is logical since at high frequencies the diffusion occurs in a small vicinity of fractures and thus is insensitive to the geometrical configuration. This is in contrast to low frequencies where penny shape fractures act essentially like 
point sources of diffusion whereas planar fractures create one-dimensional diffusion.

\subsection{Comparison With Scattering by Spherical Inclusions}

[39] The analogous scattering problem for the case of a sparse distribution of spherical inclusions in a porous medium was treated by $\mathrm{Ciz}$ et al. [2006]. The attenuation curve for the case of identical material parameters and fluid filled cavities is also shown in Figure 3. Note that the dependence on frequency is identical for the high and low frequency asymptotic behavior, only the magnitude is different. This is due to the fact that a thin crack is more compliant to deformation than a sphere, and therefore there is always going to be more fluid flow induced attenuation taking place in the medium with cracks. At low frequencies the curves are quite close, implying that at these wavelengths the differences between a sphere and a crack of the same radius do not have a great effect on the attenuation, as both are equivalent to point scatterers. At higher frequencies however there is a larger difference between spherical and crack-like inclusions as wavelengths are small enough for the shape differences to become significant.

\section{Conclusions}

[40] When the effect of a distribution of cracks is considered, assumptions regarding the interaction between individual cracks control how realistic the model is. We estimate the effect of a distribution of cracks using the multiple-scattering theorem of Waterman and Truell [1961]. This theory accounts for the interaction between scatterers (i.e., the multiple scattering) as long as the cracks are not too close together (small crack density). This analysis yields an effective velocity and attenuation due to fluid flow between pores and fractures. In the low-frequency limit, when the crack size is small compared with the fluid diffusion length, the wave period is sufficient for pressure equilibration between cracks and pores, and the velocity is consistent with the anisotropic Gassmann equations. Conversely, at high frequencies, the fluid does not have sufficient time to undergo significant flow, the cracks behave as if they were isolated from the adjacent pores, and the velocity is equal to that in the host porous medium without cracks. Attenuation has a maximum at an intermediate frequency where the diffusion length is on the order of the crack size. At low frequencies $\omega$, attenuation is proportional to $\omega$, while at high frequencies it scales with $\omega^{-1 / 2}$. This behavior is qualitatively consistent with that of infinite planar cracks in a poroelastic medium, and of scattering by spherical inclusions in such media. The theoretical model presented here can be extended to higher crack concentrations by using a self-consistent approach [Berryman, 1980a, 1980b; O'Connell and Budiansky, 1974; Zhang and Achenbach, 1991].

[41] The attenuation and dispersion due to fractures has important implications for quantitative interpretation of seismic data in fractured reservoirs. In particular, our results can be used to infer fracture size from seismic data in crosswell configuration. Furthermore, the model presented here can be used to model frequency-dependent anisotropy, which can be observed by measuring frequency-dependent shear wave splitting in VSP data. In summary, modeling of attenuation and dispersion caused by fractures can help in the characterization of fractures, host rocks and fluids.

\section{Appendix A: Far-Field Scattering Amplitude}

[42] We require $f(0)$, the far-field scattering amplitude of the fast compressional wave for a single crack in the direction of the incident wave. Since the incident wave approaches along the negative $z$ axis,

$$
f(0)=\lim _{z \rightarrow \infty} u_{z}^{1}
$$

where $u_{z}^{1}$ is given by the fast-wave $(i=1)$ term in equation (36)

$$
f(0)=\lim _{z \rightarrow \infty}\left[-\frac{\alpha}{L} \int_{0}^{\infty} A_{1}(y) q_{1} y e^{-q_{1} z} d y\right] .
$$

Because $q_{1}=\sqrt{y^{2}-k_{1}^{2}}$ is real or imaginary depending on the magnitude of $y$, it is convenient to split the integral in equation (A2) into two:

$$
\begin{aligned}
\int_{0}^{\infty} A_{1}(y) q_{1} y e^{-q_{1} z} d y= & \int_{0}^{k_{1}} A_{1}(y) q_{1} y e^{i z\left(k_{1}^{2}-y^{2}\right)^{1 / 2}} d y \\
& +\int_{k_{1}}^{\infty} A_{1}(y) q_{1} y e^{-z\left(y^{2}-k_{1}^{2}\right)^{1 / 2}} d y .
\end{aligned}
$$

Due to the presence of the negative exponential term, the second integral goes to zero as $z \rightarrow \infty$ and equation (A2) reduces to

$$
f(0)=\lim _{z \rightarrow \infty}\left[-\frac{\alpha}{L} \int_{0}^{k_{1}} A_{1}(y) q_{1} y e^{i z\left(k_{1}^{2}-y^{2}\right)^{1 / 2}} d y\right] .
$$

We can evaluate this limit using a result from Erdelyi [1956], page 47:

$$
\lim _{z \rightarrow \infty} \int_{\alpha}^{\beta} e^{i z t} \varphi(t) d t=\frac{\varphi(\beta) e^{i z \beta}}{i z}-\frac{\varphi(\alpha) e^{i z \alpha}}{i z} .
$$

Employing the substitution of variables $t=-\left(k_{1}^{2}-y^{2}\right)^{1 / 2}$ in equation (A4) reduces it to the form of equation (A5) and gives the result

$$
f(0)=\frac{\alpha k_{1}^{2}}{L} A_{1}(0)
$$

which upon substituting for $A_{1}$ using relation (28) yields equation (37).

[43] Acknowledgments. Minerals and Energy Research Institute of Western Australia, Australian Petroleum Production and Exploration Association (K.A. Richards Memorial Scholarship), Australian Research Council (Project DP0342998), and American Association of Petroleum Geologists (Sherman A. Wengerd Memorial Grant) for financial support.

\section{References}

Auriault, J.-L., and C. Boutin (1994), Deformable porous media with double porosity: III. Acoustics, Transp. Porous Med., 14, 143-162.

Berryman, J. G. (1980a), Long-wavelength propagation in composite elastic media: I. Spherical inclusions, J. Acoust. Soc. Am., 68, 1809-1819. 
Berryman, J. G. (1980b), Long-wavelength propagation in composite elastic media: II. Ellipsoidal inclusions, J. Acoust. Soc. Am., 68, 1809-1819.

Berryman, J. G., and H. F. Wang (1995), The elastic coefficients of doubleporosity models for fluid transport in jointed rock, J. Geophys. Res., 100(B12), 24,611-24,627.

Biot, M. A. (1956), Theory of propagation of elastic waves in a fluidsaturated porous solid: I. Low-frequency range, J. Acoust. Soc. Am., $28,168-178$

Biot, M. A. (1962), Mechanics of deformation and acoustic propagation in porous media, J. Appl. Phys., 33, 1482-1498.

Biot, M. A., and D. G. Willis (1957), The elastic co-efficients of the theory of consolidation, J. App. Mech., 24, 594-601.

Brajanovski, M., B. Gurevich, and M. Schoenberg (2005), A model for $\mathrm{P}$-wave attenuation and dispersion in a porous medium permeated by aligned fractures, Geophys. J. Int., 163, 372-384.

Brajanovski, M., T. M. Müller, and B. Gurevich (2006), Characteristic frequencies of seismic attenuation due to wave-induced fluid flow in fractured porous media, Geophys. J. Int., 166, 574-578.

Brown, L., and B. Gurevich (2004), Frequency-dependent seismic anisotropy of porous rocks with penny-shaped cracks, Explor. Geophys., 35(2) $111-115$

Brown, R. J. S., and J. Korringa (1975), On the dependence of the elastic properties of a porous rock on the compressibility of the pore fluid, Geophysics, 40, 608-616.

Cardona, R. (2002), Two theories for fluid substitution in porous rocks with aligned cracks, in 72st Annu. Int. Mtg., Soc. Explor. Geophys., Expanded Abstracts 21, pp. 173-176, doi:10.1190/1.817016.

Chapman, M. (2003), Frequency dependent anisotropy due to meso-scale fractures in the presence of equant porosity, Geophys. Prospect., 51, $369-379$

Ciz, R., B. Gurevich, and M. Markov (2006), Seismic attenuation due to wave-induced fluid flow in a porous rock with spherical heterogeneities, Geophys. J. Int., 165, 957-968.

Deresiewicz, H., and R. Skalak (1963), On uniqueness in dynamic poroelasticity, Bull. Seismol. Soc. Am., 53, 783-788.

Erdelyi, A. (1956), Asymptotic Expansions, Dover, New York.

Galvin, R. J., and B. Gurevich (2007), Scattering by a circular crack in a fluid-saturated porous medium, Int. J. Solids Struct., 44, 7389-7398.

Gassmann, F. (1951), Über die elastizität poröser medien, Vierteljahrsschr. Naturforsch. Ges. Zürich, 96, 1-23.

Gurevich, B. (2003), Elastic properties of saturated porous rocks with aligned fractures, J. Appl. Geophys., 54, 203-218.

Gurevich, B., and S. L. Lopatnikov (1995), Velocity and attenuation of elastic waves in finely layered porous rocks, Geophys. J. Int., 121, 933-947.

Gurevich, B., R. J. Galvin, M. Brajanovski, T. M. Müller, and G. Lambert (2007), Fluid substitution, dispersion and attenuation in fractured and porous reservoirs-Insights from new rock physics models, Leading Edge, 26(9), 1162-1168.
Hudson, J. A. (1980), Overall properties of a cracked solid, Math. Proc. Cambridge Philos. Soc., 88, 371-384.

Hudson, J. A. (1981), Wave speeds and attenuation of elastic waves in material containing cracks, Geophys. J. R. Astron. Soc., 64, 133-150.

Hudson, J. A., E. Liu, and S. Crampin (1996), The mechanical properties of materials with interconnected cracks and pores, Geophys. J. Int., 124 , $105-112$.

Hudson, J., T. Pointer, and E. Liu (2001), Effective-medium theories for fluid-saturated materials with aligned cracks, Geophys. Prospect., 49, $509-522$.

Jakobsen, M., T. A. Johansen, and C. McCann (2003), The acoustic signature of fluid flow in complex porous media, J. Appl. Geophys., 54, $219-246$.

Johnson, D. L. (2001), Theory of frequency dependent acoustics in patchysaturated porous media, J. Acoust. Soc. Am., 110, 682-694.

Lambert, G., B. Gurevich, and M. Brajanovski (2006), Attenuation and dispersion of p-waves in porous rocks with planar fractures: Comparison of theory and numerical simulations, Geophysics, 71, N41-N45.

Maultzsch, S., M. Chapman, E. Liu, and X. Li (2003), Modelling frequency-dependent seismic anisotropy in fluid-saturated rock with aligned fractures: Implication of fracture size estimation from anisotropic measurements, Geophys. Prospect., 51, 381-392.

Müller, T. M., and B. Gurevich (2005), Wave-induced fluid flow in random porous media: Attenuation and dispersion of elastic waves, J. Acoust. Soc. Am., 117, 2732-2741.

Norris, A. N. (1993), Low-frequency dispersion and attenuation in partially saturated rocks, J. Acoust. Soc. Am., 94, 359-370.

O’Connell, R. J., and B. Budiansky (1974), Seismic velocities in dry and saturated cracked solids, J. Geophys. Res., 79, 5412-5426.

Robertson, I. A. (1967), Diffraction of a plane longitudinal wave by a penny-shaped crack, Proc. Cambridge Philos. Soc., 63, 229-238.

Schoenberg, M., and J. Douma (1988), Elastic-wave propagation in media with parallel fractures and aligned cracks, Geophys. Prospect., 36, $571-$ 590

Thomsen, L. (1995), Elastic anisotropy due to aligned cracks in porous rock, Geophys. Prospect., 43, 805-829.

Waterman, P. C., and R. Truell (1961), Multiple scattering of waves, J. Math. Phys., 2, 512-537.

White, J. E. (1975), Computed seismic speeds and attenuation in rocks with partial gas saturation, Geophysics, 40, 224-232.

Zhang, C., and J. D. Achenbach (1991), Effective wave velocity and attenuation in a material with distributed penny-shaped cracks, Int. J. Solids Struct., 27, 751-767.

R. J. Galvin, CSIRO Division of Petroleum Resources, ARRC, 26 Dick Perry Avenue, Kensington, Perth, WA 6151, Australia. (robert.galvin@ csiro.au)

B. Gurevich, Curtin University of Technology, GPO Box U1987, Perth, WA 6845, Australia. (b.gurevich@curtin.edu.au) 